\title{
BMJ Open A cluster randomised controlled trial of a brief couple-focused psychoeducational intervention to prevent common postnatal mental disorders among women: study protocol
}

\author{
Heather Rowe, ${ }^{1}$ Karen Wynter, ${ }^{1}$ Paula Lorgelly, ${ }^{2}$ Lisa H Amir, ${ }^{3}$ \\ Sanjeeva Ranasinha, ${ }^{4}$ Jenny Proimos, ${ }^{5}$ Warren Cann, ${ }^{6}$ Harriet Hiscock, ${ }^{7}$ \\ Jordana Bayer, ${ }^{8}$ Joanna Burns, ${ }^{1}$ Jemimah Ride, ${ }^{2}$ Irene Bobevski, ${ }^{1}$ Jane Fisher ${ }^{1}$
}

To cite: Rowe $\mathrm{H}$, Wynter $\mathrm{K}$, Lorgelly $\mathrm{P}$, et al. A cluster randomised controlled trial of a brief couple-focused psychoeducational intervention to prevent common postnatal mental disorders among women: study protocol. BMJ Open 2014:4:e006436.

doi:10.1136/bmjopen-2014006436

- Prepublication history for this paper is available online. To view these files please visit the journal online (http://dx.doi.org/10.1136/ bmjopen-2014-006436)

Received 21 August 2014 Accepted 1 September 2014

\section{CrossMark}

For numbered affiliations see end of article.

Correspondence to Dr Heather Rowe; heather. rowe@mnash.edu

\section{ABSTRACT}

Introduction: Postnatal common mental disorders among women are an important public health problem internationally. Interventions to prevent postnatal depression have had limited success. What Were We Thinking (WWWT) is a structured, gender-informed, psychoeducational group programme for parents and their first infant that addresses two modifiable risks to postnatal mental health. This paper describes the protocol for a cluster randomised controlled trial to test the clinical effectiveness and cost-effectiveness of WWWT when implemented in usual primary care.

Methods and analysis: 48 maternal and child health (MCH) centres from six diverse Local Government Areas, in Victoria, Australia are randomly allocated to the intervention group (usual care plus WWWT) or the control group (usual care). The required sample size is 184 women in each group. English-speaking primiparous women receiving postpartum healthcare in participating $\mathrm{MCH}$ centres complete two computerassisted telephone interviews: baseline at 4 weeks and outcome at 6 months postpartum. Women attending intervention $\mathrm{MCH}$ centres are invited to attend $W W W T$ in addition to usual care. The primary outcome is meeting Diagnostic and Statistical Manual-IV (DSM-IV) diagnostic criteria for major depressive episode; generalised anxiety disorder; panic disorder with or without agoraphobia, agoraphobia with or without panic, social phobia, adult separation anxiety or adjustment disorder with depressed mood, anxiety or mixed depressed mood and anxiety within the past 30 days at 6 months postpartum. Secondary outcomes are self-rated general and emotional health, infant sleep problems, method of infant feeding, quality of mother-infant relationship and intimate partner relationship, and healthcare costs and outcomes.

Ethics and dissemination: Approval to conduct the study has been granted. A comprehensive dissemination plan has been devised.

Trial registration number: Australian New Zealand Clinical Trials Registry ACTRN12613000506796.

UTN: U1111-1125-8208.

\section{Strengths and limitations of this study}

- Extensive preparatory work supported by Government department responsible for the maternal and child health service delivery.

- Parallel cluster, prospective design.

- Will generate policy-relevant findings.

- Relatively short follow-up period.

- Challenges of implementing a standard intervention in diverse real-world settings.

\section{INTRODUCTION}

There is increasing recognition that postnatal common mental disorders among women are an important public health problem internationally. ${ }^{1}$ The term "postnatal depression' is in widespread use, but is a term that includes a range of non-psychotic mental disorders not just depression that are associated with functional disability, reduced social participation and diminished caregiving capacity. ${ }^{2}$ Anxiety and adjustment disorders are prevalent and problematic ${ }^{3}$ but less well recognised than depression. The cost of postnatal mental health problems to individuals, families, communities and the health system is considerable. ${ }^{4}$

International guidance recommends screening women for depressive symptoms in the postpartum year and referring them for treatment. ${ }^{5}$ However, a comprehensive approach to mental health service delivery must include the prevention of mental disorders. ${ }^{6}$ Many existing interventions are secondary prevention, and include indicated (for women with current symptoms) or selective (for women exposed to known risk factors) approaches. ${ }^{7}$ However, primary prevention strategies which are offered universally to 
reduce population prevalence are more cost-effective, less stigmatising and more likely to be used. ${ }^{8} 9$

Seven rigorously-conducted trials of universal postnatal primary prevention interventions have been published. ${ }^{10}$ Of these, only two reported beneficial effects.

Most of these interventions assessed only depression as an outcome. None of the trials stratified the outcome analyses by psychiatric history; and most did not identify the risk factor they were seeking to address. ${ }^{11}$ The What Were We Thinking (WWWT) programme was designed to address these gaps.

\section{The WWWT prevention programme}

WWWT is a brief, structured, couple-focused psychoeducational intervention to address modifiable risks and thereby prevent common postnatal mental disorders among women. It is designed to be integrated into universal postnatal primary care as part of a parenting programme.

\section{WWWT conceptual model}

The early postpartum period for a woman is characterised by reduced participation in activities outside the domestic sphere, disruption to social support in paid workplaces and social networks, and therefore greater dependence on her intimate partner. The quality of day-to-day interactions between partners is central to emotional well-being. The workload of caring for an infant and managing a household in which an infant lives is large and never complete. A partner who recognises and affirms a woman's mothering skills and understands her predicament enhances her confidence and well-being. ${ }^{11}$ Conversely a woman whose partner does not recognise or is critical of her work can feel demeaned and shamed. Brown and Harris ${ }^{12}$ articulated a social theory of depression in which such experiences of entrapment and humiliation are defined as key risks.

Caring for a highly dependent infant is isolating and repetitive, and can be anxiety-arousing, especially if the infant is unsettled and is difficult to sooth. Repeated attempts to sooth a baby who cries inconsolably or is difficult to breastfeed can be experienced as demoralising. The use of unsustainable and ineffective strategies to settle the infant to sleep can lead to cycles of increased anxiety and diminished confidence in parents. ${ }^{11}$

We propose that the impact of unsettled infant behaviour and difficulties in the partner relationship are mediated by occupational fatigue. The work of household management and infant care is neither dignified with the language of work nor accorded the occupational protections that exist in paid workplaces. Since infant care and household tasks are not named as work they can be under-recognised and trivialised. Extreme fatigue is widespread among new mothers and can have a profound negative impact on functioning. ${ }^{13}$ Unsettled infant behaviour exacerbates fatigue at this life stage and contributes to relationship difficulties. Fatigue may precede rather than be symptomatic of depression in women. $^{14}$

We postulate that depression and anxiety among mothers of infants can be conceptualised as reflecting poorly functioning intimate relationships with the partner and baby, which are potentially modifiable. Since the common mental disorders (anxiety, adjustment disorders and depression) are not easily distinguished, WWWT addresses them together.

$W W W T$ incorporates the three key elements of psychoeducation: information, skills development and emotional support. ${ }^{15}$ It uses adult learning, small group instruction and social cognition theories. It is positioned as meeting new parents' high learning needs for life stage-specific information and skills to underpin behaviour change. ${ }^{11} W W W T$ includes partners and provides skills to modify day-to-day interactions in this relationship. It includes the infant and addresses infant behaviours. It is informed by established theories and intended to be implemented in primary care.

\section{Preliminary evidence of efficacy of WWWT}

The acceptability, salience and preliminary evidence of efficacy of WWWT were demonstrated in a before-and-after controlled study. ${ }^{16}$ Control and intervention groups were recruited and followed sequentially. All received usual postpartum primary healthcare and intervention group participants were also invited to attend a $W W W T$ programme, facilitated by trained, supervised, specialist child and family health nurses.

Among women without a psychiatric history, being in the intervention group was associated with significantly reduced odds $(\mathrm{OR}=0.43,95 \%$ CI 0.21 to 0.89$)$ for a Diagnostic and Statistical Manual-IV (DSM-IV) diagnosis of depression or anxiety or adjustment disorder with depressed mood, anxiety or mixed anxiety and depressed mood in the first 6 months postpartum compared to the control group after adjusting for baseline group differences. The WWWT intervention appears to be a promising means of preventing common mental disorders. The aim of this study is to test WWWT effectiveness in a cluster randomised study when it is offered by trained nurses in their local settings.

\section{Study hypotheses}

Compared with usual care, participation by primiparous women in WWWT will by 6 months postpartum be associated with:

Hypothesis 1.1 (H1.1): lower 30-day prevalence of DSM-IV diagnoses of depressive, anxiety or adjustment disorders;

Hypothesis 1.2 (H1.2): better self-rated physical and emotional health;

Hypothesis 2 (H2): lower proportion of infants with unsettled behaviour;

Hypothesis 3 (H3): no differences in proportions of breastfed infants;

Hypothesis 4 (H4): similar mother-infant relationship; 
Hypothesis 5 (H5): greater satisfaction with the intimate partner relationship;

Hypothesis 6 (H6): more appropriate healthcare resource use, lower total healthcare costs and improved health-related quality of life, and thus will be cost-effective.

\section{METHODS AND ANALYSIS \\ Setting}

In the Australian state of Victoria in 2012 there were 77405 births, approximately 40\% (31000) of which were to nulliparous women ${ }^{17}$ and more than $99 \%$ in hospital. After hospital discharge, primary postnatal healthcare in Victoria is provided by the Maternal and Child Health (MCH) Service, which operates under the jurisdiction of the Victorian Government Department of Education and Early Childhood Development (DEECD). The service offers universal fee-free service to families with children aged from birth to 5 years. ${ }^{18}$ The service is funded jointly by the Victorian State and Local Governments $^{19}$ and operates according to practice guidelines. ${ }^{18}$ The Key Ages and Stages (KAS) Service Activity Framework describes the evidence-based content of 10 scheduled visits in the first year of a child's life and, for parents with first babies, a schedule of First Time Parent Groups (FTPs). ${ }^{20}$ FTPs are facilitated by $\mathrm{MCH}$ nurses and are intended to enhance parental emotional well-being and parent-child interaction, and to increase parental confidence, independence in child rearing and social support. ${ }^{21}$ All women giving birth in Victoria are allocated based on their residential address by their Local Government Area (LGA) to receive postnatal care at a specific $\mathrm{MCH}$ centre.

\section{Study reference group}

All aspects of the study will be overseen during weekly meeting of the study team. Progress will be reported regularly to the study reference group, which consists of all named investigators. The reference group will monitor conduct of the trial and provide expert advice at periodic teleconferences and in-person meetings.

\section{Study design}

CONSORT-compliant parallel group cluster randomised controlled trial, using the $\mathrm{MCH}$ centre as the unit of randomisation.

\section{The intervention}

The WWWT programme

The WWWT programme is implemented by trained $\mathrm{MCH}$ nurse facilitators in a single day, $6 \mathrm{~h}$ session designed for groups of 5-7 families, each consisting of mother, partner (or other caregiver) and their infant(s). Content is delivered in a variety of formats, including didactic presentations, discussion, individual and couple learning activities, practical demonstration and individual practice. A folder contains programme content, which uses attractive images and non-psychiatric language, and includes worksheets that are used during the programme and taken home by participants for later reference. The programme has 15 modules, grouped into two sections: About Babies and About Parents. ${ }^{11}$

Training and supervision of MCH staff WWWT facilitator training programme

MCH nurse-facilitators complete a training programme, which is informed by nurses' identified training needs and preferences and knowledge translation theory. ${ }^{22}$

The $W W W T$ training programme has three components. The online active learning module covers background theory, the level 1 face-to-face workshop promotes specific $W W W T$ skills and the level 2 workshop develops advanced knowledge and practical skills for facilitating $W W W T$ sessions.

\section{Facilitator's guide and equipment}

The facilitation guide is a detailed manual outlining introduction, precise content, proposed timings, learning strategies, responses to frequently asked questions and conclusions for each learning activity. Each facilitator is provided with a copy of the Guide, a baby sized doll and a muslin wrap of recommended dimensions to demonstrate wrapping and patting techniques during $W W W T$ sessions.

\section{Supportive supervision}

Supportive telephone or email supervision is provided to facilitators by the developers of WWWT or research staff after each programme delivery. Programme implementation, contextual factors and reflections on opportunities for improvement are discussed.

\section{Selection of local government areas}

The 31 LGAs in the greater metropolitan area of Melbourne are ranked by the Socioeconomic Indexes for Areas (SEIFA) Index of Relative Advantage and Disadvantage $^{23}$ and divided into high tertile, middle tertile and low tertile. Using an established sampling technique,${ }^{24}$ two LGAs were selected from each tertile and invited to participate. LGA invitations will continue until six LGAs have signed formal agreements with Monash University, which specify the study-related responsibilities of each party.

\section{Selection of MCH centres}

MCH centres that provide care for at least 16 primiparous women per annum are eligible. Exclusion criteria include a high proportion of clients from non-English speaking backgrounds and staff who are working in more than one $\mathrm{MCH}$ centre and therefore potentially in both intervention and control centres. Eight $\mathrm{MCH}$ centres will be selected randomly from the list of those meeting eligibility criteria from each LGA to yield 48 MCH centres for the trial. 


\section{Randomisation of $\mathrm{MCH}$ centres}

MCH centres will be allocated randomly in a 1:1 ratio to intervention or control arms by an independent statistician, using Stata V.12 random allocation program, ${ }^{25}$ stratifying by LGA ( figure 1).

\section{Participants}

\section{Inclusion criteria}

All primiparous women who have given birth in the preceding 2 weeks and are residing in participating LGAs and receiving postpartum healthcare in a participating $\mathrm{MCH}$ centre are eligible to participate.

\section{Exclusion criteria}

Women whose English language proficiency is insufficient to complete structured telephone interviews are not eligible to participate.

\section{Recruitment and participation}

In Victoria, birth notifications are distributed to LGAs. To comply with Victorian privacy legislation, a designated LGA officer telephones all potentially eligible women within 2 weeks of discharge from a maternity hospital to determine that they have given birth to their first baby. The officer explains that the LGA is a partner in a study investigating sleep parenting and relationships
Figure 1 Flow chart of selection and randomisation of maternal and child health centres and enrolment and follow-up of participants.

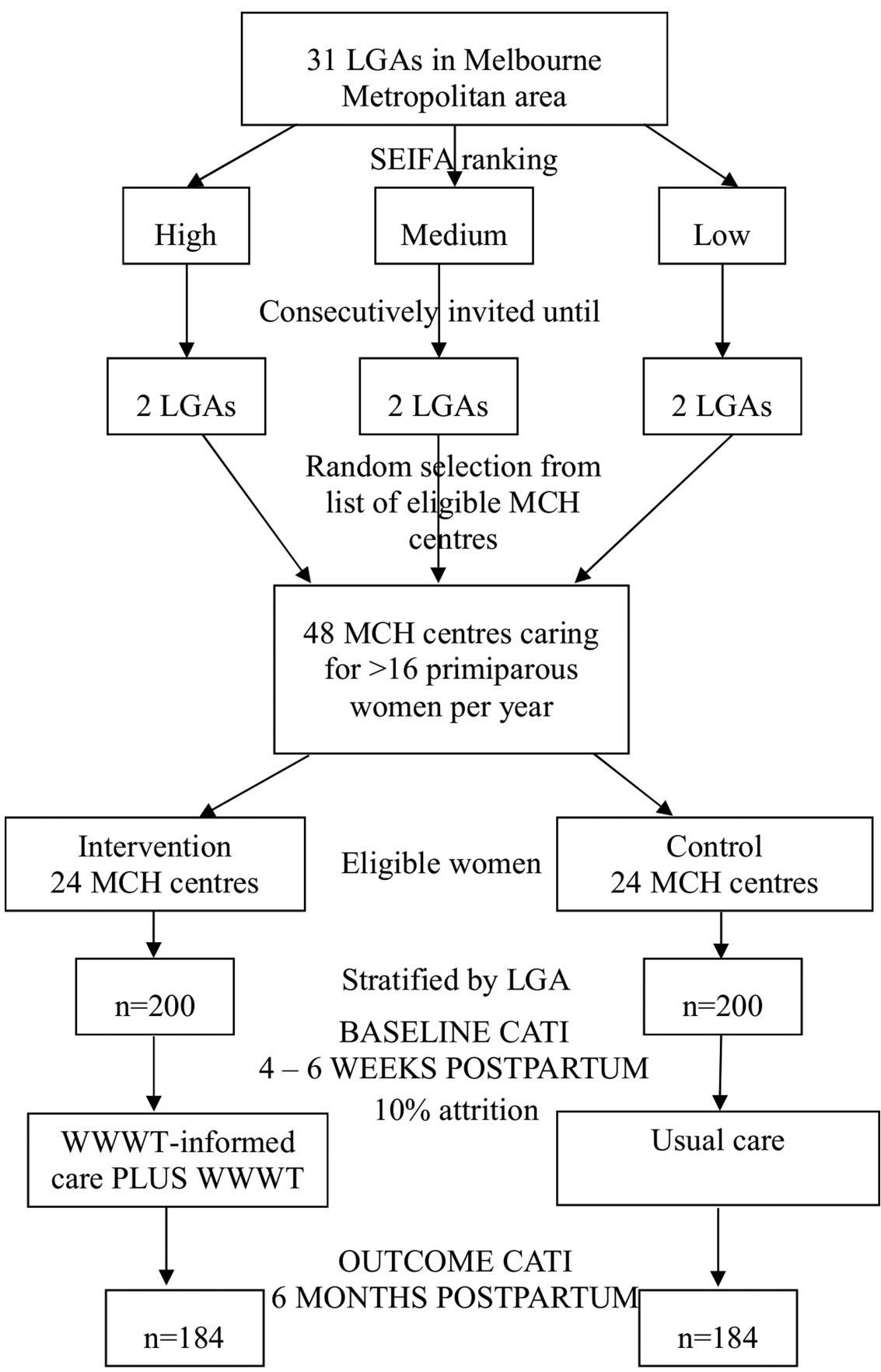


in the community and seeks an expression of interest to hear about the study. Women who are interested are asked to give permission for their contact details to be sent to the research team. Within 1 week a member of the research team telephones women who have provided their contact details to describe the study, answer questions, confirm eligibility and invite eligible women to participate. Recruitment is staged, sequentially in each LGA, to enable sufficient numbers of participants with babies of similar age to be offered $W W W T$ as a group. Recruitment will continue until the required number of participants has entered the trial.

\section{Informed consent}

The central ethical concerns are voluntary participation and protection of privacy and confidentiality. Women who express interest in participating are given an oral explanation of the study and a description of what participation would entail and are mailed or emailed the Participant Information and Consent Form. They are assured that participation is voluntary, that care providers will not know whether or not they are participating and that participation will not impact on their healthcare. Steps to safeguard participants' privacy and confidentiality are outlined. The information that participants provide will be identified only by code number, only nominated staff will have access to the data, and only pooled data from which no individual could be identified will ever be published. Women who agree to participate sign the consent form, and email or post it back to the researchers.

\section{Intervention group}

All primiparous women attending for care in intervention $\mathrm{MCH}$ centres including those recruited to the trial receive care from a WWWT-trained $\mathrm{MCH}$ nurse that is consistent with $W W W T$ principles about infant behaviour management and adjustments in the partner relationship. They are also invited to attend a WWWT programme, facilitated by trained $\mathrm{MCH}$ staff, within the first 10 weeks postpartum. They may also participate in a First-Time Parent Group, which addresses parenting topics but does not include $W W W T^{21}$

Attendance at the $W W W T$ programme will be recorded by MCH nurse facilitators on a study-specific attendance form. The lists will be used to assess participation in the intervention. Families who do not attend will be mailed a copy of the $W W W T$ participant materials.

\section{Usual care}

Participants attending control MCH centres receive usual best practice care. This may include participation in a First-Time Parent Group, but does not include participation in the $W W W T$ programme or access to the $W W W T$ print materials.

\section{Participant assessment}

After participants have given informed consent to participate, the interviewers schedule a convenient time to conduct a computer-assisted telephone interview (CATI). Follow-up is conducted at 6 months postpartum. Text messages (SMS) alert participants to the forthcoming interview and inform them that an interviewer will be contacting them. Interviewers make up to five repeat telephone calls at which time an SMS is sent informing participants that the study team will not be making further attempts to contact them. In order to promote retention, a study newsletter will be sent to participants part-way through the trial.

\section{Data Sources}

Baseline and follow-up assessments comprise studyspecific structured, CATIs. Interview instruments are available from the first author. Interviews are conducted by experienced graduate health research interviewers. Training of interviewers involves them observing trained interviewers conducting interviews and then undertaking supervised interviews themselves. Once they are proficient, interviewers begin study interviews and then participate with the researchers and other interviewers in weekly supervision and feedback sessions.

Data will be collected in the CATI platform. The CATI platform uses field limiters which minimise mis-entry and ensure that there are no missing data. CATI data are downloaded regularly to the Monash University network which is compliant with data security requirements. Only named researchers will have access to the password-protected data files.

\section{Baseline CATI}

The baseline CATI includes study-specific fixed-choice questions about the participant's date of birth, country of birth, main language spoken at home, current marital status, occupation prior to having the infant, highest level of education completed, history of diagnosed mental health problems and history of physical and sexual abuse. Study-specific questions also collect information about previous pregnancies. Questions about this pregnancy include conception using Assisted Reproductive Technology (ART), unplanned pregnancy, mode of birth, singleton infant or multiples, self-rated general health, maternal rating of infant general health and method of feeding the infant.

Participants also complete the Vulnerable Personality Style Questionnaire (VPSQ), ${ }^{26}$ Patient Health Questionnaire (PHQ-9), ${ }^{27}$ Generalised Anxiety Disorder (GAD-7), ${ }^{28}$ PHQ Panic $^{29}$; Demoralisation Scale, ${ }^{30}$ Intimate Bonds Measure (IBM) ${ }^{31}$ and a modified form of the Barr Parental Diary ${ }^{32}$ (table 1).

\section{Follow-up CATI}

\section{Primary outcome}

The primary outcome is a DSM-IV ${ }^{33}$ diagnosis of any depressive, anxiety or adjustment disorder.

The Composite International Diagnostic Interview (CIDI) yields diagnoses of anxiety and mood disorders but not of adjustment disorders. ${ }^{34}$ Thus the primary 
Table 1 Standardised instruments and psychometric properties

\begin{tabular}{|c|c|c|}
\hline Variable & Measure & $\begin{array}{l}\text { Assessment } \\
\text { time }\end{array}$ \\
\hline Personality & $\begin{array}{l}\text { Vulnerable Personality Style Questionnaire (VPSQ) }{ }^{26} \text { includes Vulnerability subscale ( } 6 \text { items) which } \\
\text { measures over-sensitivity to the opinions of others and lack of assertiveness. Internal consistency: } \\
\text { Cronbach's } \alpha=0.77 \text { and test-retest reliability: } 0.82 \text {. In a model predicting postnatal depression, sensitivity } \\
\text { was } 0.14 \text { and specificity } 0.94\end{array}$ & 1 \\
\hline Quality of relationship with intimate partner & $\begin{array}{l}\text { Intimate Bonds Measure (IBM) })^{31} \text { has two subscales: } \\
\text { Care ( } 12 \text { items) assesses sensitivity, warmth, emotional responsiveness, trust, physical gentleness and } \\
\text { kindness. Cronbach's } \alpha=0.94 \text {; correlation with clinical interview ratings of quality of relationship }=0.68 \text {. } \\
\text { Control ( } 12 \text { items) assesses coercion, dominance, exertion of power and extent of criticism. Cronbach's } \\
\alpha=0.89 \text { and correlation with clinical interview ratings of quality of relationship }=0.74\end{array}$ & 1 \\
\hline Mental disorders $(\mathrm{H} 1.1)$ & $\begin{array}{l}\text { The Composite International Diagnostic Interview v3.0 (CIDI) }{ }^{41} \text { Depression, Generalised Anxiety Disorder, } \\
\text { Panic Disorder, Agoraphobia, Social Phobia and Adult Separation Anxiety modules. The CIDI is a } \\
\text { comprehensive, standardised structured interview which can be administered by non-clinicians and yields } \\
\text { DSM-IV diagnoses. }{ }^{33} \text { It has moderate to good individual-level concordance with clinician-administered } \\
\text { DSM-IV diagnoses }{ }^{42}\end{array}$ & 2 \\
\hline Self-rated general health $(\mathrm{H} 1.2)$ & $\begin{array}{l}\text { Single question from the SF- } 36^{36} \text { 'In general, would you say your health is...' Excellent/Very good/Good/ } \\
\text { Fair/Poor. Responses to this question are associated with symptoms of physical functioning and } \\
\text { well-being }\end{array}$ & 1,2 \\
\hline $\begin{array}{l}\text { Depression symptoms, anxiety symptoms } \\
\text { and panic attacks ( } \mathrm{H} 1.2)\end{array}$ & $\begin{array}{l}\text { Depression (PHQ-9), generalised anxiety disorder (GAD-7) and panic modules of the Patient Health } \\
\text { Questionnaire. Modules are based on DSM-IV criteria. } \\
\text { PHQ-9 (Depression) })^{27} \text { ( } 9 \text { items): internal consistency Cronbach's } \alpha=0.86 \text { and } 0.89 \\
\text { GAD-7 } 7^{28}(7 \text { items): internal consistency (Cronbach's } \alpha=0.92) \\
\text { PHQ panic module: one screening item and four items about panic symptoms. Sensitivity } 0.89 \text { and } \\
\text { specificity } 0.82 \text { using clinician diagnosis as a standard }{ }^{29}\end{array}$ & 1,2 \\
\hline Demoralisation $(\mathrm{H} 1.2)$ & $\begin{array}{l}\text { Demoralisation scale }{ }^{30} \text { ( } 24 \text { items) assesses the psychological construct of demoralisation, including loss } \\
\text { of meaning; dysphoria; disheartenment; helplessness; and sense of failure. The scale has good internal } \\
\text { consistency (Cronbach's } \alpha \text { for subscales range from } 0.71 \text { to } 0.89 \text { ), and convergent and divergent validity } \\
\text { with depression measures }\end{array}$ & 1,2 \\
\hline Maternal fatigue $(\mathrm{H} 1.2)$ & $\begin{array}{l}\text { Modified form of the Fatigue Assessment Scale (FAS). }{ }^{44} \text { The FAS is a brief self-report measure of the } \\
\text { physical and cognitive aspects of fatigue. The modified form }{ }^{37} \text { ( } 5 \text { items) has been adapted for use by } \\
\text { parents of young children. In a population of } 228 \text { mothers within } 12 \text { months of giving birth, internal } \\
\text { consistency: Cronbach's } \alpha=0.88^{37}\end{array}$ & 2 \\
\hline Infant crying and fussing (H2) & $\begin{array}{l}\text { Shortened version of the Barr Parental Diary }{ }^{32} \text { : Two questions which ask parents how many hours their } \\
\text { infant fussed/cried over the two } 12 \mathrm{~h} \text { periods in the previous } 24 \mathrm{~h}\end{array}$ & 1,2 \\
\hline Infant sleep (H2) & $\begin{array}{l}\text { Single question, established indicator of day and night time sleep } \\
\text { baby's sleep generally been a problem for you'? Yes/No }\end{array}$ & 2 \\
\hline Infant sleep (H2) & & 2 \\
\hline Infant feeding $(\mathrm{H} 3)$ & Six standard questions about feeding in the previous $24 \mathrm{~h}^{45}$ & 2 \\
\hline
\end{tabular}




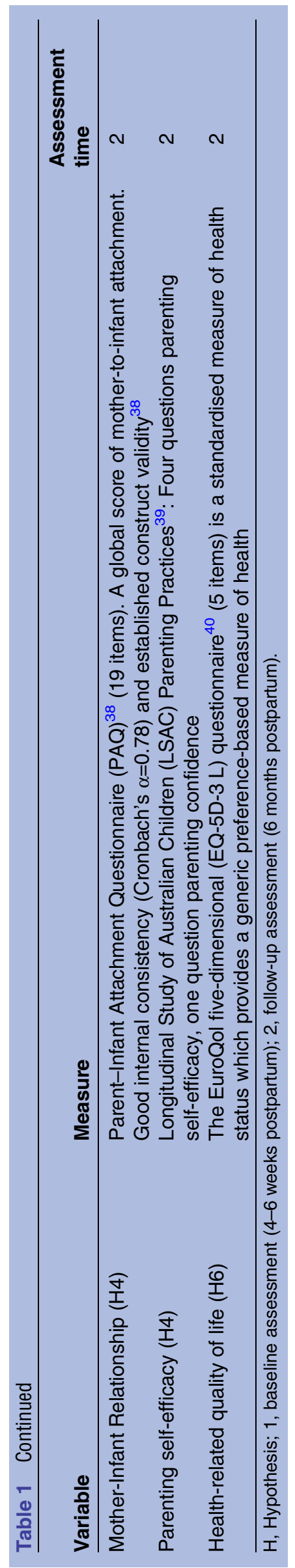

outcome (H1.1) was assessed using two separate measures and is operationalised as meeting DSM-IV ${ }^{33}$ diagnostic criteria for:

- Major depressive episode, generalised anxiety disorder, agoraphobia with or without panic, panic disorder with or without agoraphobia, social phobia or adult separation anxiety, within the past 30 days, assessed by the CIDI; ${ }^{35}$

- Adjustment disorder with depressed mood, anxiety or mixed depressed mood and anxiety are assessed using responses to symptom and impairment questions from the Patient Health Questionnaire, ${ }^{27}$ GAD- $7^{28}$ and study-specific questions about the timing of the onset of symptoms.

\section{Secondary outcomes}

Self-rated physical and emotional health (H1.2) is assessed using a single item from the SF-36, ${ }^{36}$ the $\mathrm{PHQ}^{27}$ Demoralisation Scale ${ }^{30}$ and Fatigue Assessment Scale (FAS). ${ }^{37}$

Infant sleep and unsettled infant behaviour (H2) are assessed using maternal report of an infant sleep problem, the modified form of the Barr Parental Diary ${ }^{32}$ and three questions from the Infant Feeding and Sleep Assessment Questionnaire (IFSAQ), modified for the Australian cultural context (eg, 'cot' instead of 'crib') (St James-Roberts, personal communication).

The proportion of breastfed infants (H3) is estimated using fixed-choice questions about method of infant feeding in the previous $24 \mathrm{~h}$ (breast milk, infant formula, solids.

Mother-infant relationship ( $\mathrm{H} 4)$ is assessed using the Parent-Infant Attachment Questionnaire (PAQ) ${ }^{38}$ and parenting self-efficacy questions from the Longitudinal Study of Australian Children (LSAC). ${ }^{39}$

Satisfaction with the intimate partner relationship (H5) is assessed using seven study-specific questions about perceptions of the relationship and equality of household workload distribution.

Health service use and costs (H6) are estimated from study-specific questions about healthcare resource use and out-of-pocket costs associated with postnatal healthcare for the 6 month period since the birth of the baby. To assess health-related quality of life, participants also complete the EuroQol five-dimensional questionnaire (EQ-5D-3L; ${ }^{40}$ table 1).

\section{Additional questions}

The follow-up CATI also includes study-specific questions about current marital status, unmet needs for practical and emotional support, desire for more children and an open-ended question about coincidental stressful life events. On completion of the interview, for the intervention group only, questions are asked about how often participants and their partners refer to the materials and apply the strategies from WWWT. Participants are asked to record on a study-specific form all healthcare use and out-of-pocket costs incurred since the baseline 
interview. Additional fixed-choice questions assess mastitis (breast infection with fever) and the main reason for stopping breastfeeding if relevant.

\section{Blinding}

The LGA officers who assist with recruitment of participants and the participants are blind to the allocation status of MCH centres.

The $W W W T$ programme is presented to participants as part of standard care in their MCH centre; participants will therefore be blinded to their trial status. However, there is the possibility that they will make the association between the trial investigators and the authors of the $W W W T$ programme materials.

Clinic staff are aware of the allocation status of their $\mathrm{MCH}$ centres because the WWWT programme is integrated into usual care in these MCH centres. To avoid contamination of the control MCH centres with the $W W W T$ programme, MCH staff members are asked at the Facilitator Training workshops to avoid discussion with their colleagues in control MCH centres about the $W W W T$ programme materials, training and delivery, for the duration of the trial.

The interviewers who collect baseline and outcome data are blind to allocation status until the end of the follow-up CATI when a set of questions about participation in the $W W W T$ programme is programmed to appear for those allocated to this condition.

\section{Primary outcome}

The primary outcome is assessed at the individual level at 6 months postpartum as having met DSM-IV diagnostic criteria for major depressive episode, generalised anxiety disorder, agoraphobia with or without panic, panic disorder with or without agoraphobia, social phobia or adult separation anxiety in the previous 30 days, assessed by the CIDI or adjustment disorder with anxiety or with depressed mood or with mixed anxiety and depressed mood, assessed by the PHQ.

\section{Sample size}

Based on our controlled before-and-after study we have powered the trial to detect a difference of $12.5 \%(25 \%$ in the control arm, $12.5 \%$ in the intervention arm) in prevalence of the primary outcome. A trial in which individuals are randomised would require 168 participants in each arm, with a type 1 error of $5 \%$ and $80 \%$ power. Adjusting for clustering (intraclass correlation $=0.01$ ), the required sample is $\mathrm{n}=184$ per arm, a total sample of $n=368$. Allowing for attrition of approximately $10 \%$ after baseline assessments, we will continue recruiting until 400 (200 in each arm) participants have entered the trial. With a conservatively estimated $50 \%$ recruitment fraction, the required number of participants will be able to be recruited from $48 \mathrm{MCH}$ centres (clusters), which each provide care to at least 16 primiparous women per annum (figure 1).

\section{Analysis plan}

Baseline data will be compared between the intervention and control groups to establish the validity of the randomisation process. Binary outcomes will be analysed using logistic regression models to compare the proportions at 6 months between the intervention and control groups. Continuously-valued outcomes will be analysed using linear regression and ordinal outcomes will be analysed using multinomial logistic regression. All regression analyses will be adjusted for clustering, any baseline differences, psychiatric history and other relevant covariates. In addition to the proportion of infants receiving only breast milk and any breast milk at 6 months, we will compare the proportion of women reporting mastitis and breastfeeding cessation because of low milk supply in intervention and control groups. All analyses will be by intention to treat and conducted using Stata. ${ }^{25}$

\section{Economic analysis}

The protocol for the economic evaluation being conducted with this trial is reported separately. ${ }^{46}$

\section{DATA MONITORING COMMITTEE}

A data monitoring committee is considered non-essential because the trial is conducted in a community based and not a high-risk population; the intervention being tested involves a brief one-off attendance at a WWWT session; any potential harmful effects of the intervention are assessed as unlikely; there are no preplanned interim analyses and a short follow-up of participants. ${ }^{47}$

\section{ETHICS AND DISSEMINATION}

Approval to conduct the study was granted by Southern Health (now Monash Health) Human Research Ethics Committee (24 April 2013; 11388B). The study was registered with Monash University Human Research Ethics Committee (30 April 2013; CF12/1022-2012000474). The Education and Policy Research Committee, Victorian Government Department of Education and Early Childhood Development approved the study (22 March 2012; 2012_001472). Use of the EuroQol was registered with the EuroQol Group; 16 August 2012.

Protocols are in place to identify and follow-up participants whose scores on standardised mental health assessments indicate elevated psychological distress.

A summary of results of the study in non-technical language will be posted or emailed to participants who elect to receive one. The results will be published in the peer-reviewed literature and presented to professional audiences at national and international conferences. Policy briefs will be prepared for state and local government stakeholders. Media releases will be distributed in order to make the findings known to the general public.

\section{Access to data}

The trial data set will be stored on the Monash University secure network. Only named investigators will 
have access to the data. Data will be held according to National Health and Medical Research Council policy and retained until 7 years after the results of the research are published. Access to data subsets by third parties will be negotiated according to a written data access policy and administered by the chief investigators. There are no contractual agreements that limit access.

The project lead (JF) will be responsible for final decisions about authorship. Eligibility for authorship shall be in accordance with the Australian Code for the Responsible Conduct of Research, 2007. ${ }^{48}$

\author{
Author affiliations \\ 'Jean Hailes Research Unit, School of Public Health and Preventive Medicine, \\ Monash University, Melbourne, Victoria, Australia \\ ${ }^{2}$ Faculty of Business and Economics, Centre for Health Economics, Monash \\ University, Clayton, Victoria, Australia \\ ${ }^{3}$ Judith Lumley Centre, La Trobe University, Melbourne, Victoria, Australia \\ ${ }^{4}$ Monash Centre for Health Research and Implementation, School of Public \\ Health and Preventive Medicine, Monash University, Clayton, Victoria, \\ Australia \\ ${ }^{5}$ Department of Education and Early Childhood Development, Melbourne, \\ Victoria, Australia \\ ${ }^{6}$ Parenting Research Centre, East Melbourne, Victoria, Australia \\ ${ }^{7}$ Centre for Community Child Health, The Royal Children's Hospital, Parkville, \\ Victoria, Australia; Murdoch Childrens Research Institute, Parkville, Victoria, \\ Australia; Department of Paediatrics, University of Melbourne, Parkville, \\ Victoria, Australia, Parkville, Victoria, Australia \\ ${ }^{8}$ School of Psychology, Faculty of Science, Technology and Engineering, La \\ Trobe University, Bundoora, Victoria, Australia
}

Acknowledgements The authors are grateful to the Ms Nicola Quin, Victorian Government Department of Health, Ms Ann Colahan, Victoria Government Department of Education and Early Childhood Development, Ms Helen Rowe, the Municipal Association of Victoria and staff in Local Governments who facilitated partnerships with state and local government stakeholders.

Contributors JF and HR developed the WWWT program and conducted the preliminary efficacy study. JF, HR, PL, LHA, SR, JP, WC, HH and JBa designed the trial and secured funding. $\mathrm{HR}, \mathrm{JF}, \mathrm{KW}$ and JBu drafted the manuscript and all authors reviewed the manuscript for intellectual content and approved the final version.

Funding The Victorian Government Departments of Health and Education and Early Childhood Development 2011-2012 provided a grant to assess current $\mathrm{MCH}$ practice, staff training needs and to develop a comprehensive model of costs and health and social outcomes. The trial is being funded by the National Health and Medical Research Council Project Grant APP1026550 2012-2014 and the Australian Government. JF is supported by a Monash Professorial Fellowship and the Jean Hailes Professorial Fellowship.

Ethics approval Southern Health (now Monash Health) Human Research Ethics Committee.

Provenance and peer review Not commissioned; peer reviewed for ethical and funding approval prior to submission.

Open Access This is an Open Access article distributed in accordance with the Creative Commons Attribution Non Commercial (CC BY-NC 4.0) license, which permits others to distribute, remix, adapt, build upon this work noncommercially, and license their derivative works on different terms, provided the original work is properly cited and the use is non-commercial. See: http:// creativecommons.org/licenses/by-nc/4.0/

\section{REFERENCES}

1. Gavin NI, Gaynes BN, Lohr KN, et al. Perinatal depression: a systematic review of prevalence and incidence. Obstet Gynecol 2005; 106:1071-83.
2. Brockington I. Postpartum psychiatric disorders. Lancet $2004 ; 363: 8$.

3. Ross L, McLean L. Anxiety disorders during pregnancy and the postpartum period: a systematic review. J Clin Psychiatry 2006;67:14.

4. Deloitte Access Economics. The cost of perinatal depression in Australia: final report. Postal and Antenatal Depression Association. Secondary The cost of perinatal depression in Australia: final report. Postal and Antenatal Depression Association 2012. http://www. panda.org.au/images/stories/PDFs/PANDA_Exec_Summ_Deloitte_ Web.pdf

5. National Institute for Health and Clinical Excellence. Antenatal and postnatal mental health. Cinical Management and Service Guidance. NICE clinical guidline 45 2007; guidance.nice.org.uk/cg45.

6. Herrman H, Saxena S, Moodie R, et al. Introduction: promoting mental health as a public health priority. In: Herrman $\mathrm{H}$, Saxena S, Moodie R, eds. Promoting mental health: concepts, emerging evidence, practice. Geneva: World Health Organization, 2005.

7. Mrazek P, Haggerty R. Reducing risks for mental disorders: frontiers for preventive intervention research. Washington, DC: National Academy Press, 1994.

8. Rose G. Mental disorder and the strategies of prevention. Psychol Med 1993;23:553-5.

9. McCarthy M, McMahon C. Acceptance and experience of treatment for postnatal depression in a community mental health setting. Health Care Women Int 2008;29:618-37.

10. Dennis C, Dowswell T. Psychosocial and psychological interventions for preventing postpartum depression (Review). The Cochrane Library 2013; Issue 2. doi:10.1002/14651858.CD001134.pub3/pdf/ standard.

11. Rowe H, Fisher J. Development of a universal psycho-educational intervention to prevent common postpartum mental disorders in primiparous women: a multiple method approach BMC Public Health 2010;10:499.

12. Brown GW, Harris T. The social origins of depression. A study of psychiatric disorder in women. London: Tavistock Publications, 1978.

13. Milligan R, Lenz ER, Parks PL, et al. Postpartum fatigue: clarifying a concept. Sch Inq Nurs Pract 1996;10:279-91.

14. Lam P, Hiscock H, Wake M. Outcomes of infant sleep problems: a longitudinal study of sleep, behavior, and maternal well-being. Pediatrics 2003;111:e203-7.

15. Lukens EP, McFarlane WR. Psychoeducation as evidence-based practice: considerations for practice, research, and policy. Brief Treat Crisis Interv 2004;4:205-25.

16. Fisher J, Wynter K, Rowe H. Innovative psycho-educational program to prevent common postpartum mental disorders in primiparous women: a before and after controlled study. BMC Public Health 2010;10:432.

17. Australian Bureau of Statistics. Births, Australia, 2012. Canberra; Cat 3301.02013.

18. Victorian Government Department of Education and Early Childhood Development. Maternal and Child Health Service Guidelines Secondary Maternal and Child Health Service Guidelines 2009 http://www.education.vic.gov.au/Documents/childhood/professionals/ health/mchsguidelines.pdf

19. Victorian Government Department of Education and Early Childhood Development. Memorandum of Understanding Between DEECD and MAV in Relation to Maternal and Child Health Services July 2012June 2015 Secondary Memorandum of Understanding Between DEECD and MAV in Relation to Maternal and Child Health Services July 2012-June 2015 2012. http://www.education.vic.gov.au/ childhood/professionals/health/Pages/mchpolicy.aspx\#H2N1002A

20. Victorian State Government Department of Education and Early Childhood Development and Municipal Association of Victoria. Maternal and Child Health Service: Key Ages and Stages Framework. Secondary Maternal and Child Health Service: Key Ages and Stages Framework 2009. http://www.education.vic.gov.au/ Documents/childhood/professionals/health/mchkasframework.pdf

21. Victorian Government Department of Education and Early Childhood Development. First Time Parent Group Resource and Facilitation Guide for Maternal and Child Health Nurses. Secondary First Time Parent Group Resource and Facilitation Guide for Maternal and Child Health Nurses 2001. http://www.education.vic.gov.au/Documents/ childhood/professionals/support/parentgroupguidemch.pdf

22. Wynter K, Rowe $\mathrm{H}$, Burns J, et al. Implementation of what were we thinking (WWWT) in Victorian maternal and child health services: a mixed methods investigation of acceptability, feasibility and resource implications. Melbourne: Jean Hailes Research Unit, School of Public Health and Preventive Medicine, Monash University, 2013.

23. Australian Bureau of Statistics. An introduction to socio-economic indexes for areas (SEIFA) 2006. Australian Bureau of Statistics, 2008. 
24. Hiscock H, Bayer J, Gold L, et al. Improving infant sleep and maternal mental health: a cluster randomised trial. Arch Dis Child 2007;92:952-8.

25. StataCorp. Stata Statistical Software: Release 12. College Station, TX: StataCorp LP, 2011.

26. Boyce P, Hickey A, Gilchrist J, et al. The development of a brief personality scale to measure vulnerability to postnatal depression. Arch Womens Ment Health 2001;3:147-53.

27. Spitzer RL, Kroenke K, Williams JBW. Validation and utility of a self-report version of PRIME-MD: the PHQ primary care study. JAMA 1999;282:1737-44.

28. Spitzer RL, Kroenke K, Williams JBW, et al. A brief measure for assessing generalized anxiety disorder: the GAD-7. Arch Intern Med 2006;166:1092-97.

29. Kroenke K, Spitzer RL, Williams JBW, et al. Anxiety disorders in primary care: prevalence, impairment, comorbidity, and detection. Ann Intern Med 2007;146:317-25.

30. Kissane DW, Wein S, Love A, et al. The demoralisation scale: a report of its development and preliminary validation. J Palliat Care 2004;20:269-76.

31. Wilhelm K, Parker G. The development of a measure of intimate bonds. Psychol Med 1988;18:225-34.

32. Barr R, Kramer M, Boisjoly C, et al. Parental diary of infant cry and fuss behaviour. Arch Dis Child 1988;63:380-7.

33. American Psychiatric Association. DSM-IV-TR. Diagnostic and statistical manual of mental disorders. 4th edn. Text revision. Arlington, VA: American Psychiatric Association, 2000.

34. Casey P, Bailey S. Adjustment disorders: the state of the art. World Psychiatry 2011;10:11-18.

35. World Health Organization. Composite international diagnostic interview-auto version 2.1: administrators guide and reference. Sydney: Training and Reference Centre for WHO CIDI, 1997.

36. Ware J, Snow K, Kosinski M, et al. SF-36® Health survey manual and interpretation guide. Boston, MA: New England Medical Center, The Health Institute, 1993.

37. Giallo R, Wade C, Cooklin A, et al. Assessment of maternal fatigue and depression in the postpartum period: support for two separate constructs. J Reprod Infant Psychol 2011;29:69-80.
38. Condon J, Corkindale C. The assessment of parent-to-infant attachment: development of a self-report questionnaire instrument. J Reprod Infant Psychol 1998;16:57-76.

39. Australian Institute of Family Studies. The Longitudinal Study of Australian Children Annual statistical report 2010. Secondary The Longitudinal Study of Australian Children Annual statistical report 2010. 2011. http://www.growingupinaustralia.gov.au/pubs/asr/2010/ asr2010e.html

40. Brooks R. EuroQol: the current state of play. Health Policy 1996;37:53-72

41. Kessler RC, Üstün TB. The World Mental Health (WMH) survey initiative version of the World Health Organization (WHO) Composite International Diagnostic Interview (CIDI). Int J Methods Psychiatr Res 2004:13:93-121.

42. Haro JM, Arbabzadeh-Bouchez S, Brugha TS, et al. Concordance of the Composite International Diagnostic Interview Version 3.0 (CIDI 3.0) with standardized clinical assessments in the WHO World Mental Health Surveys. Int J Methods Psychiatr Res 2006;15:167-80.

43. Schytt E, Waldenström U. Risk factors for poor self-rated health in women at 2 months and 1 year after childbirth. $J$ Womens Health 2007;16:390-405.

44. Michielsen HJ, De Vries J, Van Heck GL. Psychometric qualities of a brief self-rated fatigue measure: the Fatigue Assessment Scale. J Psychosom Res 2003;54:345-52.

45. World Health Organization. Indicators for assessing breastfeeding practices. Geneva, Switzerland: WHO, 1991.

46. Ride J, Rowe $\mathrm{H}$, Wynter $\mathrm{K}$, et al. Protocol for economic evaluation of a psycho-educational intervention for the primary prevention of postnatal mental health problems in first-time mothers, alongside a cluster-randomised controlled trial. BMJ Open 2014; in press; doi:10.1136/bmjopen-2014-006226

47. Committee for Medicinal Products for Human Use. Guideline for data monitorinig committees. London: European Medicines Agency, 2005.

48. National Health and Medical Research Council. Australian Code for the Responsible Conduct of Research. 2007. https://www.nhmrc. gov.au/guidelines/publications/r39 